PALLIATIVE CARE

\section{Why should we care about palliative care for AIDS in the era of antiretroviral therapy?}

\section{P A Selwyn}

The possibility of pharmacological interactions between HIV medications and palliative care medications makes it essential to coordinate HIV specific and palliative care

o nitially, in the now distant early years of the AIDS epidemic in the developed world, AIDS care was palliative care. As HIV/AIDS therapies have evolved rapidly since the mid-1990s, the focus of clinical care has increasingly been on antiretroviral therapy and the complex decision making that surrounds its use. While the growing "medicalisation" of AIDS is in part because of the advent of effective treatment with the possibility of controlling viral replication and disease progression, the emergence of this more biomedical paradigm has resulted in a loss of perspective on chronic disease and the issues relevant to progressive, incurable illness and end of life care. Early in the epidemic, HIV care providers were by definition palliative care providers. Now the challenge is to reacquaint what have since developed into two distinct disciplines, in order to provide our patients with the benefits of both types of expertise.

As outlined in the review article by Harding et al in this issue of STI (p 5), it is important for HIV care providers to remain sensitive to the need for palliative care interventions across the continuum of HIV disease. Palliative medicine has much to offer to HIV care, and can help improve quality of life, enhance adherence to HAART (highly active antiretroviral therapy) and other therapies, and address the complicated psychosocial issues now faced by patients with AIDS and their families. One of the unintended consequences of the HAART era has been the separation of AIDS care from palliative care: as our focus has narrowed, and our skills in reversing the previously inexorable course of HIV disease have grown, our overall care of the patient has lost some of its completeness.

Studies have consistently documented the under-recognition and undertreatment of pain and other symptoms in patients with AIDS, and have also consistently demonstrated a high prevalence of pain and chronic symptoms such as fatigue, anorexia/weight loss, nausea/vomiting, dyspnoea, and diarrhoea, both in the pre-HAART and current eras. ${ }^{1-7}$ Within the narrow biomedical model of care, such symptoms are generally regarded as secondary, or incidental, to the primacy of the infectious disease diagnosis which dictates appropriate pathogen specific treatment. While treating specific infections is obviously an essential and fundamental strategy in AIDS treatment, clinicians could benefit from the realisation that there is a large and growing evidence base for palliative medicine interventions which can be very effective as adjunctive or primary treatment for many of these disabling symptoms. ${ }^{8-10}$

In the developed world, we now face difficult decisions about withdrawal of care, goals of therapy, and acceptance of treatment failure and death in the age of HAART that are different from those in the former era when death was expected, imminent, and universal

In fact, one can argue that by treating such symptoms effectively-including, for example, the common gastrointestinal symptoms which can be induced by antiretroviral and other HIV specific therapies-we are in fact helping to promote adherence, which can be jeopardised when patients associate medication use with adverse subjective symptoms. Moreover, the possibility of pharmacological interactions between HIV medications (for example, certain antiretrovirals) and palliative care medications (for example, certain benzodiazepines, opioids, anticonvulsants, non-sedating antihistamines) makes it essential to coordinate HIV specific and palliative care. These are only two examples of how AIDS care should not be "either...or" - that is, either palliative or disease specific therapy-but rather "both...and" in the hope of integrating both paradigms in the comprehensive care of patients with AIDS. ${ }^{11}$

As the review indicates, there is also evidence that home based and community based care for patients with advanced illness can provide benefits in quality of life, and as we also know, chronic care coordination is essential for good adherence to HAART regimens over time. The HAART era has taught us that without these long term, community based systems for care and follow up, the risk of antiretroviral resistance grows, and patients are at risk of becoming "untreatable," with multiple drug resistant HIV. We would do well to learn from the field of hospice and palliative care, where the approach of coordinated, multidisciplinary, home and community based care has been followed successfully for decades.

It should also be mentioned that in addition to the benefits of palliative care for improving quality of life and, potentially, HIV treatment adherence, the need for end of life care has not disappeared in the HAART era. Approximately 15000 Americans continue to die from AIDS and/or co-existing conditions each year (for example, cirrhosis/liver failure, other end organ disease, non-AIDS defining cancers), and the rates of mortality decline have flattened since the late 1990s. HIV/AIDS remains a leading cause of death for African-Americans and Latinos, and for the foreseeable future, end of life care will be an unavoidable aspect of HIV care in these populations as well as in large areas of the developing world. In addition, in the developed world, we now face difficult decisions about withdrawal of care, goals of therapy, and acceptance of treatment failure and death in the age of HAART that are different from those in the former era when death was expected, imminent, and universal.

If anything, these matters have become more complex and nuanced than they were previously, and it is necessary for us to attend to the psychosocial issues involved in end of life care that may not be as familiar to today's new generation of HIV care providers as they were in the earlier era when death was inescapable. To turn away from these important clinical issues makes us less effective in our treatment of our patients. If we become "antiretroviral specialists" then we lose something in our ability to provide our patients with the comprehensive care they need. The science and evidence base of palliative medicine have much to offer for improvement in quality of life, relief of suffering, enhancement of disease specific therapy, expert end of 
life care, and coping with chronic, progressive illness for patients and their loved ones. Recent efforts have attempted to address the importance of integrating HIV specific and palliative care in the HAART era. ${ }^{12}$ Our patients deserve nothing less.

Sex Transm Infect 2005;81:2-3.

doi: $10.1136 /$ sti.2004.011585

Correspondence to: Dr Peter A Selwyn, Department of Family and Social Medicine Montefiore Medical Center, Albert Einstein College of Medicine, Bronx, NY 10467, USA; selwyn@aecom.yu.edu

\section{REFERENCES}

1 LaRue F, Colleau SM. Underestimation and undertreatment of pain in HIV disease: a multicentre study. $B M J$ 1997;314:23-8

2 Breitbart W, Rosenfeld B, Passik SD, et al. The undertreatment of pain in ambulatory AIDS patients. Pain 1996:65:243-9.

3 Fantoni M, Ricci F, Del Borgo C, et al. Multicentre study on the prevalence of symptoms and symptomatic treatment in HIV infection. J Palliat Care 1997:13:9-13.

4 Fontaine A, LaRue F, Lasauniere JM. Physicians; recognition of the symptoms experienced by HIV patients: how reliable? J Pain Symptom Manage 1999:18:263-70.

5 Mathews W, McCutcheon JA, Asch S, et al. National estimates of HIV-related symptom prevalence from the HIV Cost and Services Utilization Study. Med Care 2000:38:762

6 Vogl D, Rosenfeld B, Brietbart W, et al. Symptom prevalence, characteristics, and distress in AIDS outpatients. J Pain Symptom Manage 1998; 18:253-62.

7 Selwyn PA, Rivard M, Kapell D, et al. Palliative care for AIDS at a large urban teaching hospital: program description and preliminary outcomes. $J$ Palliat Med 2003;6:461-74

8 Doyle D, Hanks GWC, MacDonald N, eds. Oxford textbook of palliative medicine, 2nd ed. New York: Oxford University Press, 1998.

9 End of Life Physician Education Resource Center (EPERC). www.eperc.mcw.edu accessed 16 July, 2004

10 O'Neill J, Selwyn PA, Schietinger $\mathrm{H}$, eds. A clinical guide to supportive and palliative care for HIV/AIDS. Rockville, MD: Health Resources and Services Administration, 2003

11 Selwyn PA, Forstein M. Overcoming the false dichotomy of curative vs palliative care for late-stage HIV/AIDS: let me live the way I want to live, until I can't. JAMA 2003;290:806-14

12 Workgroup on Palliative and End-of-Life Care in HIV/AIDS. Promoting excellence in end-of-life care, a national program of the Robert Wood Johnson Foundation. Integrating palliative care into the continuum of HIV care: an agenda for change (www.promotingexcellence.org), April, 2004

\section{Sexual health and HIV in Scotland}

\section{R Nandwani}

\section{Find out more about how Scotland is tackling its poor sexual health}

is

your required knowledge of Scotland is limited to "Tartan tourism" and our

inferior national football team, you may wish to stop now. If, however, you wish to find out more about how Scotland is tackling its poor sexual health, read on.

Scotland faces similar challenges to much of the United Kingdom with high levels of unwanted conception and rising STI and HIV rates. The population of around five million is concentrated in the central belt of Scotland with relatively poor access to sexual health services in many areas. Despite this, there has been a dramatic rise in diagnosed genital chlamydia infection, especially in those under 25 years old. There were 11917 laboratory diagnoses between January and September 2004, compared with 10124 during the same period in 2003. Although some of the increases in chlamydia are related to changes in sexual behaviour as elsewhere in the United Kingdom, testing has become more easily accessible owing to developments such as the Sandyford Initiative in Glasgow and the new West Lothian outreach clinics, which both provide integrated community based services. Self testing for chlamydia has been promoted in Aberdeen and by Healthy Respect, the sexual health demonstrator project, in Lothian. Healthy Respect is

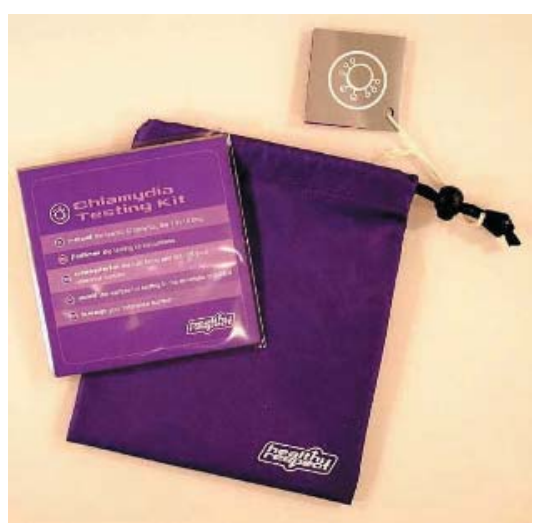

currently evaluating the impact of postal chlamydia testing. The kits (see fig 1) have been distributed in community sites such as pharmacies and record shops as well as in health settings. Other STIs including gonorrhoea continue to rise in Scotland. There is also an ongoing syphilis outbreak with a 146\% increase between 2003 and 2004, predominantly in men who have sex with men. Fast track outreach testing is being performed in Glasgow to reach homosexual men who might not otherwise use conventional settings. There are peer supported services specifically for men who have sex with men in many areas, notably the Steve Retson Project in
Glasgow and Man Friday in Grampian. There has been significant integration of genitourinary medicine with family planning and reproductive health in Scotland, which has helped establish high quality services, notably for young people but also for other socially excluded populations including the homeless, those from black and ethnic minority groups, and people with physical or learning disabilities.

Many associate HIV in Scotland with injecting drug users, given the large cohort infected in Edinburgh in 1984; however, sex between men cumulatively accounts for the largest proportion of the 4128 HIV cases diagnosed to date. Owing to the rapid implementation of needle exchanges and methadone projects, the number of new Scottish HIV cases acquired by needle sharing is now in single figures each year. Despite this, there remain a significant number of HIV positive drug users with hepatitis $\mathrm{C}$ co-infection. Recently, there has been a large rise in the number of heterosexuals infected abroad being treated in Scotland. This, coupled with the successful implementation of opt out testing in GUM clinics, has resulted in a rise in new HIV diagnoses in the past 3 years. The average in the 1990s was 150-180 new cases per year. There were 258 new HIV diagnoses in 2003 and from January to September 2004 there have already been 274 new HIV diagnoses.

BASHH Scotland is well supported and has been ably led by Dr Andy Winter in its first year. Achievements have included the delivery of a secure web based data collection for GUM clinic coding. With pump priming money from the Scottish Executive, all clinics acquired new computers and the new "STISS" (STI Surveillance Scotland) is now live. The STI Foundation course is also thriving in Scotland with 617 delegates attending the 
first 13 courses including 388 doctors ( 167 of whom were GPs) and 165 nurses. Much effort has been put into supporting sexual health workers from all disciplines, particularly in developing competencies and training. Several new genitourinary medicine consultant led services have been established in Scotland including in Paisley, Borders and Ayrshire; however, recruitment of staff is a challenge for smaller units despite the high quality of life on offer in Scotland.

By the time you read this, I hope that the Scottish Executive Health Department will have released their implementation plan and resources for the Sexual Health and Relationships Strategy. The draft strategy, produced by an expert reference group, was presented to the Scottish Executive in November 2003 and was followed by a period of public consultation. The strategy takes a broad societal approach with sections on promoting positive sexual health, education, mass communications, leadership, and accountability as well as service provision. Inevitably, this has generated headlines (often misinformed) and public debate orchestrated by those who believe that sexual activity should be confined to heterosexual marriage. The approach of BASHH Scotland has been to share best practice and ensure patient access is maintained (often by very busy drop-in services). The message given to the Scottish Executive and others is that GUM has responded positively to capacity and case mix pressures by modernising and delivering the best possible models of service, often in partnership with others. So far there has not been significant investment in Scottish GUM using either HIV cash or new funding stemming from the Sexual Health Strategy in England and Wales. Therefore, implementation of the Scottish Strategy is eagerly awaited.
ELECTRONIC INFORMATION

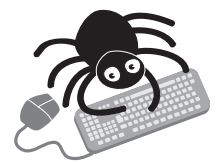

Scottish Sexual Health Strategy: http:// www.scotland.gov.uk/sexualhealthstategy; BASHH Scotland: http://www.bashh.org/ committees/regions/scotland/ (includes response to Scottish Strategy); Scottish sexual health statistics: http://www.show.scot.nhs.uk/ scieh and http://www.isdscotland.org/isd/ ; Scottish online GUM clinic data collection: http://www. show.scot.nhs.uk/sticoding/. Test version you can try out for yourself: Sandyford Initiative: http://www. sandyford.org; includes links to the Steve Retson Project, young people's service, and GP support websites; Healthy Respect: http:// www. healthy-respect.com

Sex Transm Infect 2005;81:3-4 doi: $10.1136 /$ sti.2004.014290

Correspondence to: R Nandwani, The Sandyford Initiative, Glasgow, UK; Rak.Nandwani@glacomen.scot.nhs.uk

\section{$\mathrm{ECHO}$}

\section{Gonococcal endocarditis}

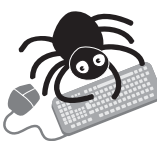

Please visit the Sexually Transmitted Infections website [www. stijournal.com] for a link to the full text of this article.
G onococcal infection is a rare cause of endocarditis but one to be borne in mind. An illustrative case has been described from Cardiff, UK.

A 28 year old man developed symptoms several days after having unprotected sexual intercourse with a partner who had been treated for genitourinary symptoms. The patient's symptoms included sore throat, lethargy, malaise, joint pains, sweating, and mainly nocturnal fevers. He was given a course of amoxycillin $500 \mathrm{mg}$ three times a day but his symptoms persisted and when next seen by his general practitioner he had developed a new, moderately loud, pansystolic murmur at the apex and radiating to the axilla. On admission to hospital finger clubbing and a vasculitic lesion of the left great toe were noted as well as the murmur. Investigations showed a neutrophil leukocytosis $\left(18 \times 10^{9} /\right.$ litre $)$, raised $\mathrm{C}$ reactive protein (>120 mg/litre), raised erythrocyte sedimentation rate $(51 \mathrm{~mm} /$ hour $)$, and low serum albumin ( $29 \mathrm{~g} /$ litre). Treatment for bacterial endocarditis (intravenous benzylpenicillin $1.2 \mathrm{~g}$ four hourly plus gentamicin $80 \mathrm{mg}$ twice daily) was begun after taking four separate sets of blood cultures. On transthoracic echocardiography there was a large ( $1.35 \mathrm{~cm}$ diameter $)$ echodense mass on the anterior mitral valve leaflet with severe mitral regurgitation.

On the fourth hospital day growths of Neisseria gonorrhoeae serotype WI were reported from three blood culture bottles. The organism had a minimum inhibitory concentration for penicillin of $0.03 \mu \mathrm{g} / \mathrm{ml}$ and was resistant to ciprofloxacin. The antibiotic treatment was not changed at that time but on day 17 the gentamicin was replaced by rifampicin $600 \mathrm{mg}$ daily because of lack of clinical response. Two days later he developed severe nausea that was attributed to the penicillin and cefotaxime $2 \mathrm{~g}$ six hourly was substituted for penicillin but he remained feverish and the markers of inflammation remained high. Mitral valve replacement was therefore performed on day 25. Intravenous cefotaxime was continued for two weeks and he was well when he left hospital on oral anticoagulant treatment 38 days after admission.

Disseminated gonococcal infection occurs in up to $3 \%$ of all patients with gonococcal infection and gonococcal endocarditis in $2 \%$ or fewer of those with disseminated infection (up to one in 1670 patients with gonorrhoea). There has, however, been an increase in the incidence of gonococcal infection in the UK in recent years and increasing rates of ciprofloxacin resistance may lead to more patients being treated inadequately and therefore being at risk of disseminated infection. Gonococcal endocarditis tends to occur in younger people (age 15-35 years) and is more common in men. The period from symptom onset to diagnosis of endocarditis averages around 45 days and is intermediate between the acute endocarditis of staphylococcal and pneumococcal infections and the subacute endocarditis of Streptococcus viridans infection. Two thirds of patients with gonococcal endocarditis have no genitourinary symptoms and only about one in eight has a history of prior valvular heart disease. More than half need valve surgery and mortality may be as high as $20 \%$.

Gonococcal endocarditis is rare but may become more common with increasing rates of gonococcal infection. Its clinical features differ from those of other types of bacterial endocarditis but a firm diagnosis will depend on blood cultures.

\ Shetty A, et al. Journal of Clinical Pathology 2004;57:780-781. 